\title{
Genome position specific priors for genomic prediction
}

\author{
Rasmus Froberg Brøndum", Guosheng Su, Mogens Sandø Lund', Philip J Bowman², Michael E Goddard²,3 \\ and Benjamin J Hayes ${ }^{2,4,5}$
}

\begin{abstract}
Background: The accuracy of genomic prediction is highly dependent on the size of the reference population. For small populations, including information from other populations could improve this accuracy. The usual strategy is to pool data from different populations; however, this has not proven as successful as hoped for with distantly related breeds. BayesRS is a novel approach to share information across populations for genomic predictions. The approach allows information to be captured even where the phase of SNP alleles and casuative mutation alleles are reversed across populations, or the actual casuative mutation is different between the populations but affects the same gene. Proportions of a four-distribution mixture for SNP effects in segments of fixed size along the genome are derived from one population and set as location specific prior proportions of distributions of SNP effects for the target population. The model was tested using dairy cattle populations of different breeds: 540 Australian Jersey bulls, 2297 Australian Holstein bulls and 5214 Nordic Holstein bulls. The traits studied were protein-, fat- and milk yield. Genotypic data was Illumina 777K SNPs, real or imputed.

Results: Results showed an increase in accuracy of up to $3.5 \%$ for the Jersey population when using BayesRS with a prior derived from Australian Holstein compared to a model without location specific priors. The increase in accuracy was however lower than was achieved when reference populations were combined to estimate SNP effects, except in the case of fat yield. The small size of the Jersey validation set meant that these improvements in accuracy were not significant using a Hotelling-Williams t-test at the 5\% level. An increase in accuracy of 1-2\% for all traits was observed in the Australian Holstein population when using a prior derived from the Nordic Holstein population compared to using no prior information. These improvements were significant $(P<0.05)$ using the Hotelling Williams t-test for protein- and fat yield.

Conclusion: For some traits the method might be advantageous compared to pooling of reference data for distantly related populations, but further investigation is needed to confirm the results. For closely related populations the method does not perform better than pooling reference data. However, it does give an increased accuracy compared to analysis based on only one reference population, without an increased computational burden. The approach described here provides a general setup for inclusion of location specific priors: the approach could be used to include biological information in genomic predictions.
\end{abstract}

Keywords: Genomic prediction combined populations, Genomic location, Bayesian prediction

\footnotetext{
* Correspondence: rasmusf.brondum@agrsci.dk

${ }^{1}$ Centre for Quantitative Genetics and Genomics, Department of Molecular Biology and Genetics, Faculty of Science and Technology, Aarhus University, Tjele 8830, Denmark

Full list of author information is available at the end of the article
}

\section{Biomed Central}

(c) 2012 Brøndum et al.; licensee BioMed Central Ltd. This is an Open Access article distributed under the terms of the Creative Commons Attribution License (http://creativecommons.org/licenses/by/2.0), which permits unrestricted use, distribution, and reproduction in any medium, provided the original work is properly cited. 


\section{Background}

Genomic predictions are now widely used in dairy cattle breeding, and have been proposed for breeding of crops and prediction of disease risk in humans $[1,2]$. The accuracy of genomic estimated breeding values, depends on a number of factors, of which the size of the reference population used to estimate the marker effects is critical [3]. In dairy cattle, the reference population usually consists of progeny-tested bulls. In small populations, such as Australian Jersey, the number of progeny-tested bulls available for the reference is limited. For genetically related populations such as the European Holstein populations and Nordic Red Cattle populations previous studies show large benefits from pooling reference populations $[4,5]$, but for more distantly related populations (e.g., Holstein and Jersey) this approach does not increase the accuracy to the same extent [6,7]. Previous studies based on Single Nucleotide Polymorphism (SNP) markers from the Illumina 50K SNP chip [8] have reported that distances between markers would be too large for high persistence of linkage disequilibrium (LD) phase across breeds, and accuracies of across breed prediction were zero $[9,10]$. With the new Illumina $777 \mathrm{~K}$ chip, it is expected that distances between markers are small enough for successful genomic prediction using combined reference data from different dairy cattle populations, as the Quantitative Trait Loci (QTL)-SNP phase in such high density markers would be well preserved across breeds [3]. However, a recent study demonstrated only limited support for this hypothesis, with relatively small gains resulting from pooling Australian Holstein and Jersey data to improve the accuracy of Jersey genomic predictions [11]. This suggests that there are still differences in the patterns of LD between single markers and actual QTL across breeds, and thus pooling the data might dilute associations of markers with phenotypic traits.

In this study, we explore an alternative approach to pooling data across breeds. Previous studies have shown that some parts of the genome explain more variation than others. Assuming that the same causative mutations, or even the same gene regions but different causative mutations, act on traits of interest in different populations, it is expected that effects of chromosome regions on a trait could be consistent among populations, though the LD patterns between individual SNPs and QTLs could differ from one population to the other. At the extreme it was demonstrated that there was considerable overlap in gene regions affecting stature in humans and cattle [12]. The aim of this study was to first map the variation explained by small segments of the bovine genome for production traits in three dairy cattle populations, and compare this variation across the populations. In particular, we explored the effect of segment size on the correlation of the variances across populations. Subsequently this information was used as genomic location specific priors in a new method for predicting genomic estimated breeding values. Developing a model with location specific prior information will also allow for differentiation between e.g. coding and non-coding regions of the genome, or other kinds of biological information.

\section{Methods \\ Data}

The datasets used in the present study included 540 Australian Jersey bulls (JER-AUS), 2297 Australian Holstein bulls (HOL-AUS) and5214 Nordic (Danish, Swedish or Finnish) Holstein bulls (HOL-NOR) (Table 1). Phenotypic data was daughter trait deviations (DTD) for the Australian bulls and deregressed proofs (DRP) for the Nordic bulls. DTDs were given in kilograms, whereas the DRPs are standardized indices. The traits selected for the study were: protein yield, fat yield and milk yield, as these traits have the most data in the populations.

Genotypic data was a mixture of true and imputed SNP markers from the Illumina $777 \mathrm{~K}$ SNP chip. For HOL-AUS there were 843 Holstein heifers genotyped on the 777K SNP chip as well as 93 key ancestor bulls. For JER-AUS 93 key ancestor bulls were genotyped for the 777K SNP chip. Quality control steps included removing SNPs with very low minor allele frequencies, ambiguous or undefined map positions, and no heterozygote genotypes. For full details see [11]. These animals were used as reference to impute the high density genotypes for the remaining 2204 Holstein and 447 Jersey bulls which were genotyped with the $50 \mathrm{k}$ chip.

For HOL-NOR 557 bulls from the EuroGenomics project [4] were genotyped using the $777 \mathrm{~K}$ chip and these bulls were used as reference to impute the $777 \mathrm{~K}$ markers for the bulls genotyped with the Illumina 50K SNP chip. After imputation LD of each marker with the previous one in the assembly was inspected. If two adjacent markers were in complete LD one of the markers was deleted, so that $\mathrm{r}^{2}$ of any pair of adjacent markers was less

\section{Table 1 Data overview}

\begin{tabular}{llllcl}
\hline Breed & N ref & $\mathbf{N}$ val & N total & N markers & Phenotype \\
\hline JER-AUS & 454 & 86 & $\mathbf{5 4 0}$ & 465542 & DTD \\
\hline HOL-AUS & 1897 & 360 & $\mathbf{2 1 9 7}$ & 465542 & DTD \\
\hline HOL-NOR & $\mathbf{3 0 4 7}$ & 2167 & 5214 & 465542 & DRP \\
\hline
\end{tabular}

The populations used for the analysis, the number of animals in the reference ( $\mathrm{N}$ ref) and in the validation set ( $\mathrm{N}$ val), total number of animals ( $\mathrm{N}$ total), number of markers ( $\mathrm{N}$ markers) and type of response variable (Phenotype) used in the prediction models. The sets of animals used for estimation of segment variances are highlighted in bold. 
than one. The marker data was further edited by deleting markers with a minor allele frequency less than 0.01 .

Imputation was done using Beagle [13] in all three populations. Since the purpose was to compare segments across populations and use this information for genomic prediction, the SNP datasets were further edited to only keep the markers that were in common across the populations. After data editing 465,542 markers remained for analysis.

Each of the datasets was split into a reference and validation set (Table 1) to allow for cross validation of the accuracy of DGV. In HOL-NOR the bulls were separated by birth date before or after 2001-10-01, and in JER-AUS and HOL-AUS the bulls were split by onset of progeny test before or after 2007. In both cases the younger animals were assigned to the validation set. This cross validation strategy was chosen as the resulting accuracy is the most meaningful in the context in which the genomic predictions will be used: in the dairy industry. Here reference sets of older bulls are used to predict the DGV for young bulls which are then selected for use based on these DGV. In the Australian data all of the available data was used to estimate segment variances to maximize the data. In the Nordic dataset only the reference set was used to estimate segment variances.

All genotypic and phenotypic data was obtained from pre-existing routine genetic evaluation data for the dairy cattle populations and required no ethical approval.

\section{Estimation of genetic variances explained by different segments}

Genetic variance attributed to each segment was estimated from effects of the SNPs in the segment. Prediction of single SNP marker effects was carried out using BayesR [11]. The statistical model was:

$$
\boldsymbol{y}=\mu \mathbf{1}+\boldsymbol{W g}+\boldsymbol{a}+\boldsymbol{e}
$$

Where $\mathbf{y}$ is the vector of DTD or DRP, $\mu$ is the mean, $\mathbf{a}$ is the vector of residual polygenic effects, $\mathbf{e}$ is the vector of the residual errors, $\mathbf{W}$ is the incidence matrix of SNP genotype coefficients, and $\mathbf{g}$ is the vector of SNP allele substitution effects. Let $\mathbf{X}$ be a genotype matrix coded as $0 / 1 / 2$, for respectively homozygote first allele, heterozygote and homozygote second allele, and let $\mathrm{p}_{j}$ be the frequency of the second allele at locus $j$. Assuming Hardy-Weinberg equilibrium, entries of $\mathbf{W}$ are then obtained by centering and standardizing entries of $\mathbf{X}$ to:

$$
w_{i j}=\frac{x_{i j}-2 p_{j}}{\sqrt{2 p_{j}\left(1-p_{j}\right)}}
$$

Prior distributions for the parameters are given as:

$$
\begin{aligned}
& g_{j} \mid \sigma_{j}^{2} \sim N\left(0, \sigma_{j}^{2}\right) \\
& \sigma_{j}^{2}=\left\{\begin{array}{c}
0 * \sigma_{g}^{2} \text { with probability } \pi_{0} \\
0.0001 * \sigma_{g}^{2} \text { with probability } \pi_{1} \\
0.001 * \sigma_{g}^{2} \text { with probability } \pi_{2} \\
0.01 * \sigma_{g}^{2} \text { with probability } \pi_{3}
\end{array}\right. \\
& \sigma_{g}^{2}=r_{y}^{2} * \operatorname{Var}(\boldsymbol{y}) \\
& \boldsymbol{\pi} \sim \operatorname{Dirichlet}(1,1,1,1) \\
& \boldsymbol{e} \sim N\left(\mathbf{0}, \boldsymbol{I} \sigma_{e}^{2}\right) \\
& \boldsymbol{a} \sim N\left(\mathbf{0}, \boldsymbol{A} \sigma_{a}^{2}\right) \\
& \sigma_{e}^{2}, \sigma_{a}^{2}, \mu \propto 1
\end{aligned}
$$

Where $\mathbf{A}$ is the additive relationship matrix, $\sigma_{\mathrm{a}}^{2}$ is the variance of residual polygenic effects, and $r_{y}^{2}$ is the reliability of DRP/DTD. The four-distribution mixture chosen for the SNP effects, does not reflect any biological hypothesis, but was chosen to allow for easier mixing between SNPs with no effect and SNPs with effects of different sizes. The Dirichlet prior on the proportions of different SNP variances with all parameters set to one, is actually a uniform prior, but specifying it in this manner reflects the fact that the posterior distribution on the proportions follows a Dirichlet distribution with a pseudo count of 1 from each of the four distributions. The prior is not uninformative in any statistical sense since it states that all distributions have equal probabilities, but it adds very little information compared to the posterior, as the data gives information on almost half a million counts, and the prior only adds 4 , see [11] for more detail.

To estimate the variance explained by each small chromosomal region, the entire set of SNPs was divided into segments of a fixed length (e.g. 100 markers each). The variance explained by segment $\mathrm{s}$ was calculated as

$$
\operatorname{Var}\left(\boldsymbol{W}_{\mathbf{s}} \boldsymbol{g}_{s} \mid \text { data }\right)
$$

Where $\mathbf{W}_{\mathbf{s}}$ is the sub-matrix of $\mathbf{W}$ corresponding to the SNPs in segment $\mathbf{s}$, and $\mathbf{g}_{\mathbf{s}}$ is the vector of estimated SNP marker effects for the same segment, i.e. the segment variance is the variance across individuals of the partial direct genetic values (DGVs, marker only estimated breeding values) belonging to segment $\mathrm{s}$. Variances of the partial DGVs for all segments were calculated at each iteration in the Gibbs sampler, and the estimated segment variances were obtained as the posterior means. Segment variances were estimated for segment sizes of 10, 25, 50, 100, 250, $500,1000,2000$ or 3000 SNPs and for entire chromosomes. The approach is similar to [14] where sliding windows of five consecutive SNPs are used to estimate the genetic variance of chromosomal regions. In our approach the windows are however not overlapping. 
Posterior means of the parameters were obtained using a Gibbs sampler run for 20,000 iterations with a burn-in of 10,000 in the Holstein populations. For the Jersey population results were not consistent with only 20,000 iterations, so a chain length of 100,000 with a burn-in of 50,000 was used instead. The relatively poor mixing properties of the Gibbs sampler for the Jersey data could be due to the small size of the reference population. Lengths of the chains were based on preliminary runs and comparisons of the obtained segment variances. With 20.000 iterations the Holsteins showed a mean pairwise correlation between segment variances from 10 consecutive runs of 0.95 , whereas the Jerseys showed a mean correlation between segment variances from 10 consecutive runs of 0.80 . Increasing the number of iterations for the Jerseys to 100.0000 increased the mean correlation of segment variances between consecutive runs to 96 .

\section{Prediction using location specific prior information}

The purpose here is to build a Bayesian prediction model that allows for a larger proportion of variation to be explained by certain segments, based on knowledge from previous experiments. One way to do this is to allow different segments to have different prior probabilities assigned to the four SNP effect distributions. Letting $S$ denote the set of segments, the model used here is:

$$
\begin{aligned}
& \boldsymbol{y}=\mu \mathbf{1}+\sum_{s} \boldsymbol{W}_{s} \boldsymbol{g}_{s}+\boldsymbol{a}+\boldsymbol{e} \\
& g_{s j} \mid \sigma_{s j}^{2} \sim N\left(0, \sigma_{s j}^{2}\right) \\
& \sigma_{s j}^{2}=\left\{\begin{array}{c}
0 * \sigma_{g}^{2} \text { with probability } \pi_{\mathrm{s} 0} \\
0.0001 * \sigma_{g}^{2} \text { with probability } \pi_{\mathrm{s} 1} \\
0.001 * \sigma_{g}^{2} \text { with probability } \pi_{\mathrm{s} 2} \\
0.01 * \sigma_{g}^{2} \text { with probability } \pi_{\mathrm{s} 3}
\end{array}\right. \\
& \sigma_{g}^{2}=r_{y}^{2} * \operatorname{Var}(\boldsymbol{y}) \\
& \boldsymbol{\pi}_{s} \sim \operatorname{Dirichlet}\left(\boldsymbol{\alpha}_{s}\right) \\
& \boldsymbol{e} \sim N\left(0, \boldsymbol{I} \sigma_{e}^{2}\right) \\
& \boldsymbol{a} \sim N\left(0, \boldsymbol{A} \sigma_{a}^{2}\right) \\
& \sigma_{e}^{2}, \sigma_{a}^{2}, \mu \propto 1
\end{aligned}
$$

Here $\pi_{s}$ is the probability vector for the four SNP effect distributions in segment $s$, and $\alpha_{s}$ is the vector of prior parameters for the Dirichlet distribution in segment s. The model is similar to the original BayesR model, with the modification that the probability to sample SNPs from the four different distributions now depends on the segment. By setting the location specific information via the Dirichlet prior, instead of using constant proportions, the model estimates the proportions using both the data and the prior information. As this is a BayesR by segment approach, the model will be referred to as BayesRS.
To test the BayesRS model, posterior means of the number of times in which the indicator variable fell in component $i$ of the mixture were estimated in each segment in one population using BayesR, and subsequently used as the $\alpha_{\mathrm{s}}$ parameters in the target population in BayesRS. This was done for segment sizes of 100, 250, 500, 1000, 2000 or 3000 SNPs. Since the sum of counts in the alpha parameters in this setup is equal to the number of markers, this means that the prior on the proportions in the mixture, unlike in the regular BayesR, now has as much weight as the data (much higher weight than in BayesR). To test the impact of the weight of the prior, different scaling factors were tried, i. e. the $\alpha_{\mathrm{s}}$ parameters were multiplied by $0.2,0.4, .0 .6,0.8,1.0$, 1.25 or 1.5 . The model was tested in three different scenarios:

1. JER-AUS with prior information from HOL-AUS.

2. HOL-AUS with prior information from HOL-NOR.

3. HOL-AUS (random) with prior information from HOL-NOR.

HOL-AUS (random) is a random subset of 500 animals from the HOL-AUS reference population, which was generated to test the hypothesis that the advantage of the BayesRS model would be greater in smaller populations. The second and third setups were tested using the same validation animals.

\section{Validation of DGV accuracy}

DGVs for the validation populations were predicted as

$$
D G V_{\boldsymbol{k}}=\hat{\mu}+\boldsymbol{w}_{\boldsymbol{k}}^{\prime} \hat{\boldsymbol{g}}+\hat{a}_{\boldsymbol{k}}
$$

Where $w^{\prime}{ }_{k}$ is the row of $\mathbf{W}$ belonging to animal $k$. Accuracies of the DGV were calculated as r(DGV, DTD) and validated in HOL-AUS and JER-AUS. Differences in accuracy between BayesR and BayesRS were tested for significance using a Hotelling-Williams t-test, which takes account of the number of individuals in the validation set [15].

\section{Results and discussion}

The posterior means of the number of times the indicator variable fell in each of the four distributions in BayesR for all three breeds and all three traits are shown in Table 2. Results are in line with [11] where it was also found that only a small percentage of the markers have an effect. The markers with the highest posterior probability of being in the largest effect distributions are unevenly distributed across the segments, and as the table also shows large proportions of the marker variance is expected to be explained by a small number of markers, which suggest that the location specific priors add extra information.

\section{Segment variances}

Table 3 shows the top 10 segments for all three traits and all three populations for a segment size of 100 SNPs. 
Table 2 Distribution of SNP effects and proportion of expected total marker variance for each class

\begin{tabular}{|c|c|c|c|c|c|c|c|c|c|}
\hline \multirow[b]{2}{*}{ Distribution } & \multicolumn{3}{|c|}{ JER-AUS } & \multicolumn{3}{|c|}{ HOL-AUS } & \multicolumn{3}{|c|}{ HOL-NOR } \\
\hline & Protein & Fat & Milk & Protein & Fat & Milk & Protein & Fat & Milk \\
\hline 0 & $462816(0 \%)$ & $461458(0 \%)$ & $461173(0 \%)$ & $462734(0 \%)$ & $461215(0 \%)$ & $462715(0 \%)$ & $460980(0 \%)$ & $461134(0 \%)$ & $460155(0 \%)$ \\
\hline 0.0001 & $2413(35 \%)$ & $3752(45 \%)$ & 4111 (49\%) & $2623(52 \%)$ & $4223(70 \%)$ & $2487(38 \%)$ & 4387 (67\%) & $4270(64 \%)$ & $5205(65 \%)$ \\
\hline 0.001 & 299 (44\%) & $318(38 \%)$ & $239(28 \%)$ & $179(36 \%)$ & $95(16 \%)$ & $332(50 \%)$ & $170(26 \%)$ & $127(19 \%)$ & $171(21 \%)$ \\
\hline 0.01 & $14(21 \%)$ & $14(17 \%)$ & 19 (23\%) & $6(12 \%)$ & $9(15 \%)$ & $8(12 \%)$ & $5(8 \%)$ & $11(17 \%)$ & $11(14 \%)$ \\
\hline
\end{tabular}

Posterior means of the number of times the indicator variable fell in each of the four distributions in the mixture for protein yield, fat yield and milk-yield in Australian Jersey (JER-AUS), Australian Holstein (HOL-AUS) and Nordic Holstein (HOL-NOR). Expected proportion of marker variance in each class was calculated as the number of markers in the class times the proportion of genetic variance assigned to each marker $(0,0.0001,0.001$ or 0.01$)$ divided by the sum of marker variance in all classes.

Table 3 Top 10 segments ranked by proportion of variance

\begin{tabular}{|c|c|c|c|c|c|c|c|c|c|c|c|}
\hline \multicolumn{4}{|c|}{ Protein yield } & \multicolumn{4}{|c|}{ Fat yield } & \multicolumn{4}{|c|}{ Milk yield } \\
\hline Prop Var & CHR & Start & End & Prop Var & CHR & Start & End & Prop Var & CHR & Start & End \\
\hline \multicolumn{12}{|l|}{ JER-AUS } \\
\hline 2.51 & 14 & 1324152 & 2524432 & 2.63 & 14 & 1324152 & 2524432 & 9.23 & 14 & 1324152 & 2524432 \\
\hline 0.94 & 8 & 53145498 & 53823453 & 0.53 & 6 & 32804873 & 33205790 & 1.03 & 23 & 33488986 & 34003600 \\
\hline 0.49 & 29 & 32616370 & 33148216 & 0.5 & 16 & 34988324 & 35436695 & 0.88 & 20 & 17220850 & 17690967 \\
\hline 0.46 & 13 & 63320438 & 64620664 & 0.26 & 9 & 47503904 & 48177473 & 0.75 & 16 & 35920 & 804371 \\
\hline 0.38 & 23 & 36309465 & 36929103 & 0.21 & 27 & 35936818 & 36385854 & 0.45 & 23 & 39769575 & 40258674 \\
\hline 0.34 & 3 & 89581663 & 89898693 & 0.2 & 1 & 127226200 & 127917611 & 0.33 & 10 & 30761581 & 31494927 \\
\hline 0.31 & 17 & 69563223 & 70374082 & 0.18 & 22 & 60504152 & 60873210 & 0.24 & 8 & 73117701 & 73898467 \\
\hline 0.31 & 29 & 33157623 & 33719571 & 0.17 & 12 & 34663468 & 35292842 & 0.22 & 5 & 93922247 & 94302255 \\
\hline 0.3 & 29 & 34401183 & 34817726 & 0.15 & 13 & 27805120 & 28346480 & 0.2 & 20 & 28252035 & 28774228 \\
\hline 0.25 & 9 & 48742490 & 49474068 & 0.15 & 5 & 120908187 & 121179132 & 0.17 & 20 & 34452105 & 35077755 \\
\hline \multicolumn{12}{|l|}{ HOL-AUS } \\
\hline 10.38 & 14 & 1324152 & 2524432 & 17.97 & 14 & 1324152 & 2524432 & 16.05 & 14 & 1324152 & 2524432 \\
\hline 1.27 & 6 & 88537190 & 88996262 & 2.71 & 5 & 93922247 & 94302255 & 0.7 & 6 & 88537190 & 88996262 \\
\hline 0.58 & 7 & 82862759 & 83380203 & 0.34 & 5 & 93301390 & 93920010 & 0.66 & 20 & 31054019 & 31704692 \\
\hline 0.52 & 18 & 58283983 & 59602905 & 0.31 & 2 & 107799001 & 108408740 & 0.63 & 5 & 93301390 & 93920010 \\
\hline 0.48 & 3 & 117198648 & 117541474 & 0.28 & 26 & 20643699 & 21338653 & 0.46 & 18 & 33639529 & 33910770 \\
\hline 0.44 & 6 & 89469872 & 90304531 & 0.25 & 4 & 106613116 & 107060437 & 0.42 & 7 & 82862759 & 83380203 \\
\hline 0.31 & 28 & 18048845 & 18758510 & 0.18 & 25 & 8073067 & 8481205 & 0.37 & 14 & 69793328 & 70364164 \\
\hline 0.3 & 11 & 38575857 & 38932630 & 0.16 & 11 & 102944335 & 103540503 & 0.33 & 20 & 34452105 & 35077755 \\
\hline 0.2 & 1 & 136016808 & 136624372 & 0.12 & 20 & 36176136 & 36613401 & 0.31 & 25 & 14686647 & 15151658 \\
\hline 0.2 & 18 & 57084113 & 57818432 & 0.11 & 16 & 56014214 & 56706854 & 0.28 & 11 & 46783081 & 47198727 \\
\hline \multicolumn{12}{|l|}{ HOL-NOR } \\
\hline 4.85 & 14 & 1324152 & 2524432 & 24.35 & 14 & 1324152 & 2524432 & 13.49 & 14 & 1324152 & 2524432 \\
\hline 0.39 & 19 & 26550090 & 27153052 & 2.65 & 5 & 93922247 & 94302255 & 2.37 & 20 & 29983162 & 31051302 \\
\hline 0.31 & 7 & 23881292 & 24505374 & 0.76 & 19 & 20077363 & 20545023 & 0.9 & 5 & 92171816 & 92734379 \\
\hline 0.31 & 5 & 20094983 & 20608440 & 0.64 & 5 & 20094983 & 20608440 & 0.89 & 5 & 92736297 & 93292054 \\
\hline 0.28 & 6 & 86786552 & 87331055 & 0.5 & 15 & 44850860 & 45134081 & 0.63 & 5 & 20094983 & 20608440 \\
\hline 0.25 & 11 & 102944335 & 103540503 & 0.26 & 20 & 63502967 & 63925075 & 0.46 & 5 & 93922247 & 94302255 \\
\hline 0.23 & 22 & 42655109 & 43216893 & 0.25 & 19 & 26550090 & 27153052 & 0.38 & 15 & 52804974 & 53411913 \\
\hline 0.18 & 23 & 11564383 & 12095383 & 0.23 & 2 & 127612583 & 128084951 & 0.33 & 24 & 59281770 & 59735242 \\
\hline 0.16 & 24 & 59281770 & 59735242 & 0.21 & 26 & 20043160 & 20630551 & 0.33 & 6 & 88023038 & 88527916 \\
\hline 0.16 & 20 & 69275055 & 69727331 & 0.19 & 13 & 10469479 & 11785572 & 0.23 & 11 & 101054186 & 101516564 \\
\hline
\end{tabular}

Proportion of variance (Prop Var) explained for Jersey (JER-AUS), Australian Holstein (HOL-AUS) and Nordic Holstein (HOL-NOR) populations. Segment size is 100 SNPs. Start and end points of the segments are given as base positions of the first and last SNP in the segment. Segments that appear in more than one breed are highlighted in bold. 
For all traits and populations there is one segment with very large effect, located on chromosome 14 . This segment is located around the DGAT1 gene which has a well-documented effect on the traits in question [16]. Furthermore for fat percentage in the Holsteins there is a segment with large effect on chromosome 5. This segment has previously been seen in GWAS studies in Australian Holsteins and validated in Australian Jerseys [17]. The segment, however, does not appear in the top 10 for the Jersey population. In the Nordic Holstein population a segment having large effect on milk yield is found on chromosome 20, but the same segment is not found in the top 10 for either JER-AUS or HOL-AUS although other segments on chromosome 20 are present. A previous study found a growth hormone receptor gene on chromosome 20 which was highly associated with milk yield [18]. However, the same gene could not be validated in the Australian Holstein population [19], and the segments on chromosome 20 found in the top 10 in this study do not coincide with the gene in question.

Correlations of segment variances between populations are large if the DGAT1 segment is included since the proportion of variance explained for this QTL is very large compared to all others, as seen from Table 3 and Figure 1. Hence SNPs associated with the DGAT1 mutation have been removed from all plots in Figure 2 for clarity. For fat yield the QTL on chromosome 5 with large effect has been removed as well. Figure 2 shows a plot of segment size versus correlations of segment variances between the three populations for the three traits. With the large effect segments removed, the patterns of correlations generally follow expectations based on knowledge of genetic relationship between populations. Highest correlations are found between HOL-AUS and HOL-NOR, and second and third place are respectively HOL-AUS versus JER-AUS and HOL-NOR versus JER-AUS. In Australia some crossbreeding has taken place between the Holstein and Jersey populations and, accordingly, correlations of variance explained by the segments are higher for these two populations than between HOL-NOR and JER-AUS. Genotype by environment interactions could also influence these correlations.

Correlations for small segment sizes are close to zero. A possible explanation for this is that differences in LD patterns and SNP allele frequencies across breeds cause the SNPs with the highest associations to actual QTL to be placed in different segments when these are very small. The rapid increase in correlation of segment variances with segment size for HOL-AUS vs. HOL-NOR suggests that these population share QTL in similar locations, as would be expected given they are genetically closely related. For this pair of populations, even reasonably small segments would convey information between the populations. When taking an entire chromosome as a segment, correlations of segment variances ranging from 0.6 between JER-AUS and HOL-NOR up to 0.8 between the HOL-AUS and HOL-NOR were found. A connection between chromosome size and variance explained has previously been reported by e.g. [20]. As seen from Figure 3 a similar pattern can be found in our populations, provided the effect of DGAT1 is removed from the analysis.

\section{BayesRS}

The accuracies of DGV, measured as $\mathrm{r}$ (DGV,DTD) in the validation population, are shown in Figure 4 and 5 . In the figures, a horizontal line depicts the accuracy using BayesR. For JER-AUS a second (higher) horizontal line shows the accuracies obtained from BayesR when simply pooling JER-AUS with HOL-AUS. For the HOLAUS with HOL-NOR information scenario this accuracy was not available due to data sharing policies.

For JER-AUS no gain in accuracy was observed for milk yield when using prior information from $\mathrm{HOL}$ -
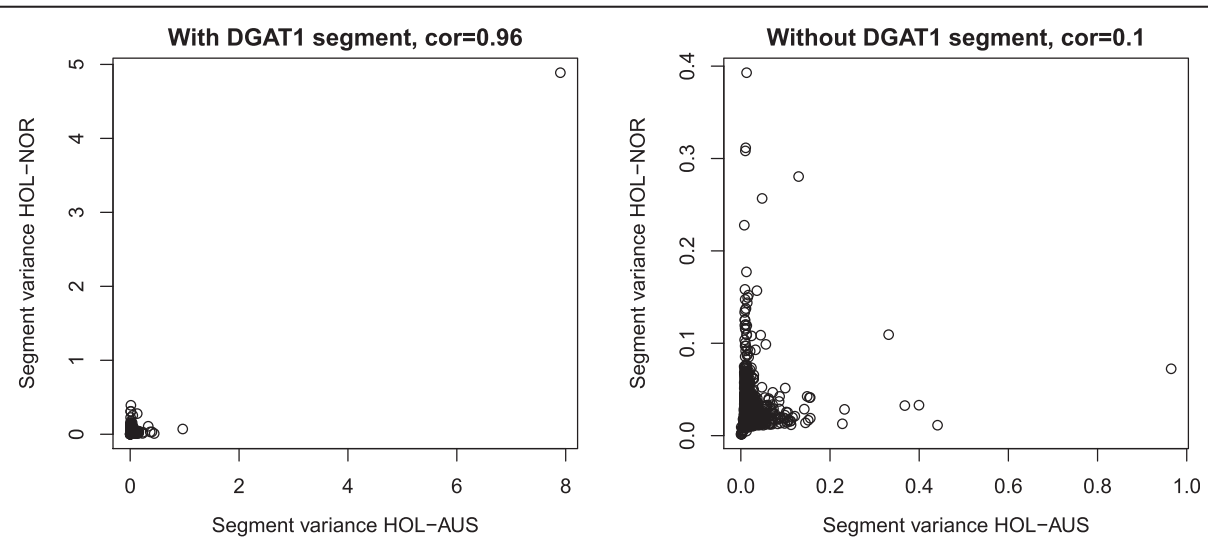

Figure 1 Comparison of segment variances between Australian Holstein (HOL-AUS) and Nordic Holsteins (HOL-NOR) with and without the effect of DGAT1, shown here for protein yield and a segment size of 100 SNPs. 

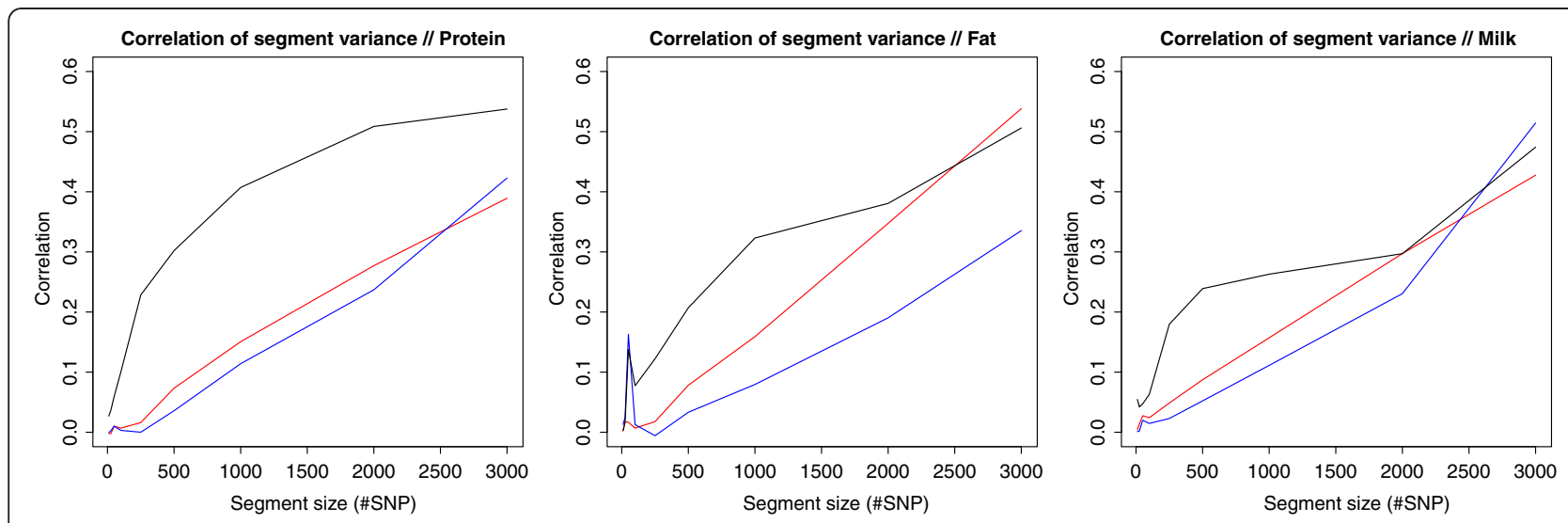

Figure 2 Correlation of segment variances for Protein-, Fat- and Milk yield between JER-AUS and HOL-AUS (red), JER-AUS and HOL-NOR (blue) and HOL-AUS and HOL-NOR (black). The segment containing DGAT1 has been removed for all traits, as has the large effect on chromosome 5 for fat yield.

AUS, for protein yield a small gain of around $1 \%$ is seen for the smallest segment size, and for fat yield gains in accuracy of up to $3.5 \%$ are seen when using the genomic location specific prior information compared to using BayesR. Compared with accuracies obtained with a simple pooling of reference data, the BayesRS approach leads to an extra gain of up to $1.5 \%$ for fat yield, but not for the other two traits. Although differences in accuracy were seen, none of the differences were significant at a $5 \%$ level, reflecting the small size of validation population. For HOL-AUS the largest gain in accuracy is found for protein yield with gains of up to $2 \%$. For milk and fat

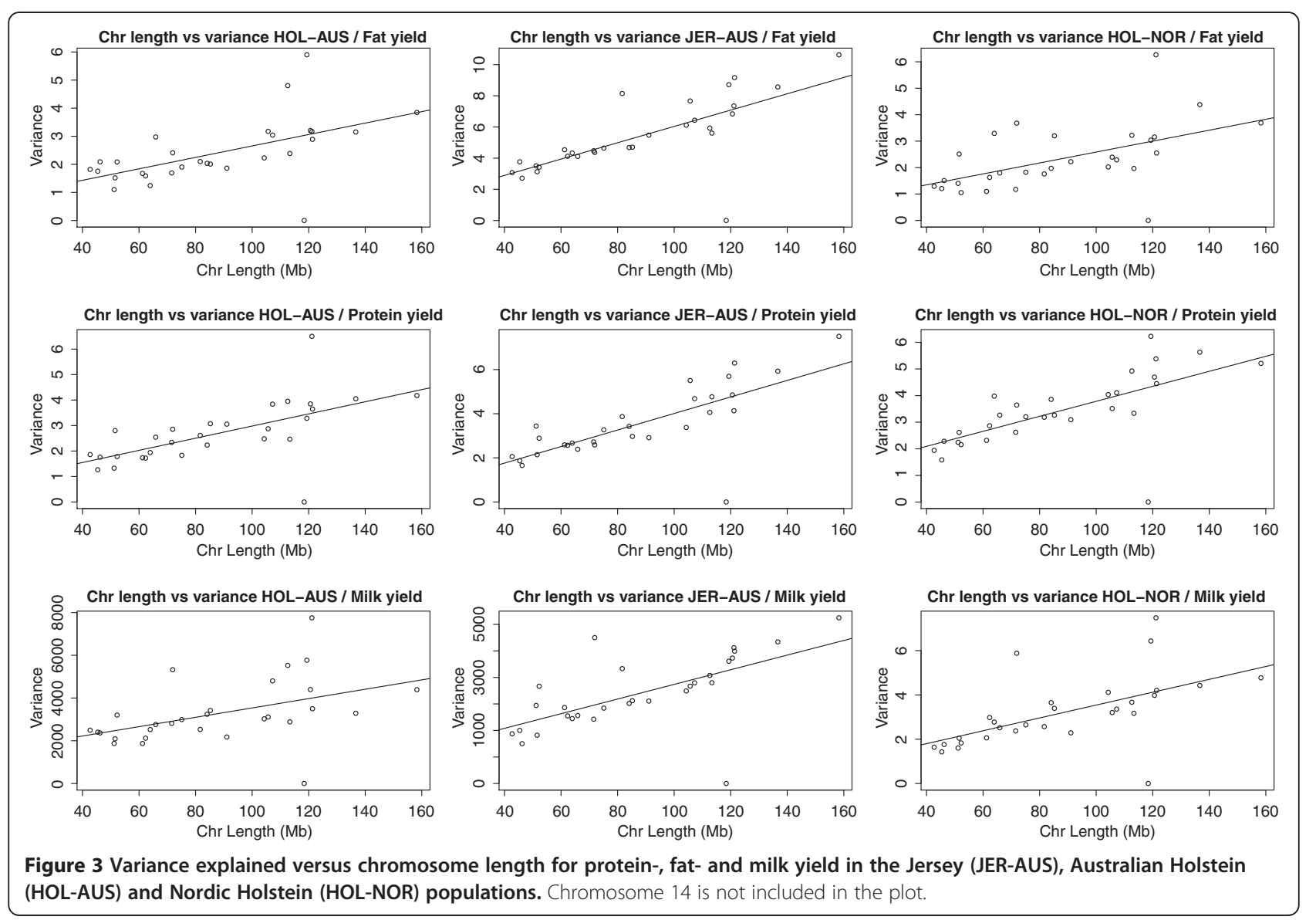




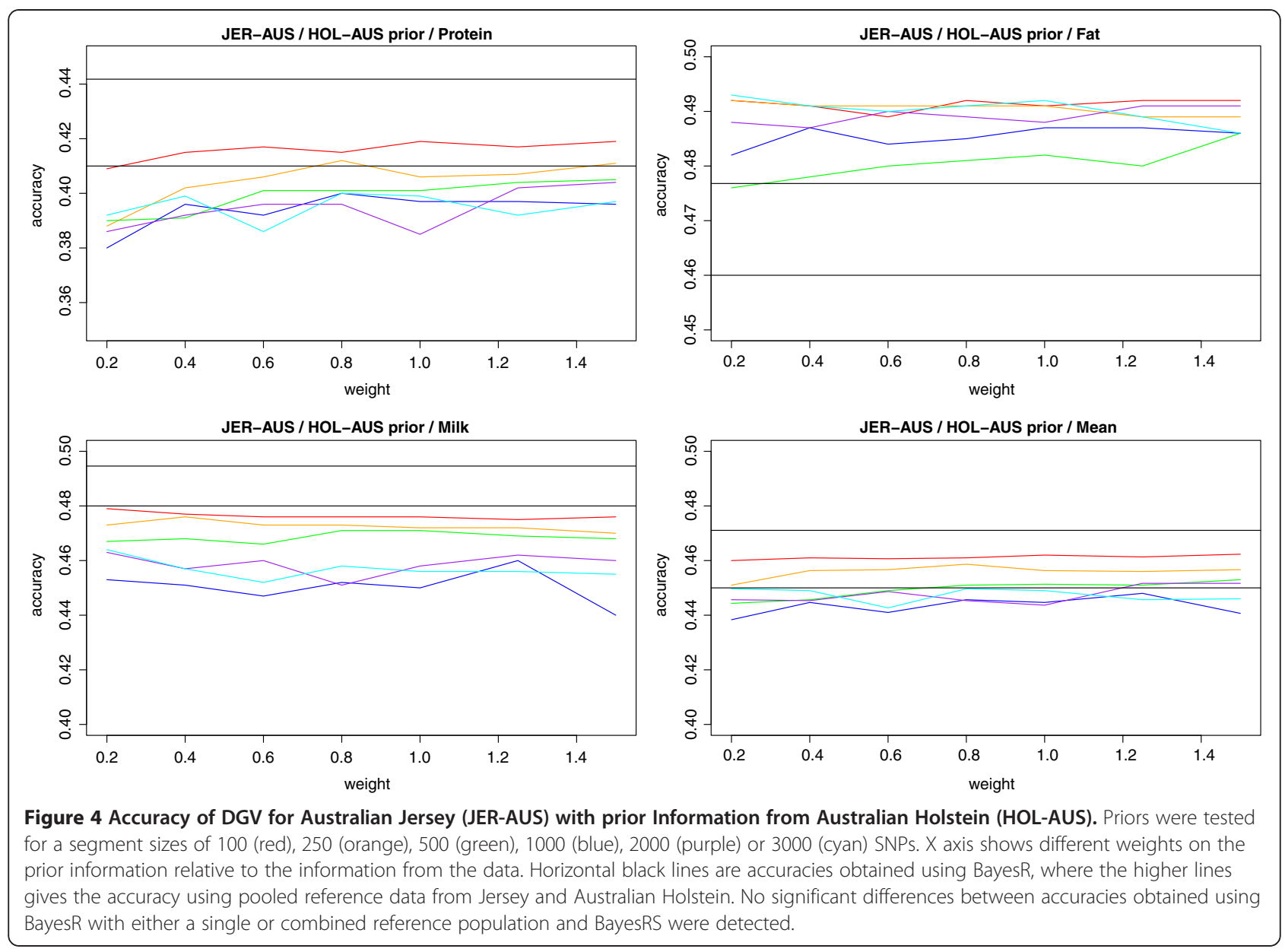

yield smaller gains are seen, and these are not significantly different for milk yield. Using the prior information derived from HOL-NOR, however, seems consistently better than the model without location specific priors. Results from the HOL-AUS (random) setup are shown in Figure 6 . The gain in accuracy is here slightly higher for protein and fat yield, whereas the gain for milk yield seems unchanged. Results were significant for protein and fat but not for milk yield. Looking at the accuracies of DGV from the regular BayesR model (no prior information from other populations) the mean accuracy is now on the same level as in JER-AUS due to the same size of the reference population (though the traits vary considerably).

In all three scenarios the highest gains in accuracy are found for a segment size of 100 markers, implying that using smaller segments gives a stronger advantage from the location specific priors. Furthermore, significant results are only found in two cases: the largest and smallest segments. For the largest segment size of 3000 markers, it is surprising that the increase in accuracy is significant although larger gains in accuracy are seen for smaller segments. However, this could be an artifact of the test chosen for the significance. With a large segment size the added information becomes very unspecific which could lead to results more similar to those obtained from the regular BayesR method. With a high correlation between DGVs from the two methods, the Hotelling Williams t-test would cause even small differences in accuracy to be significant.

The different scaling factors (weights) applied to the parameters in the Dirichlet priors, seems to make little or no difference on the accuracy of the BayesRS model, which suggests that the accuracies obtained from BayesRS could be random fluctuations. This is in many, but not all cases, supported by the lack of significance of the results.

To summarize, BayesRS gave accuracies comparable to, but not always higher than or significantly different from, a simple pooling of the data. For closely related populations pooling is expected to be superior. So a simple pooled multi-breed or multi-population reference could be a better approach in some cases, but not necessarily for all traits. For example, the BayesRS approach 


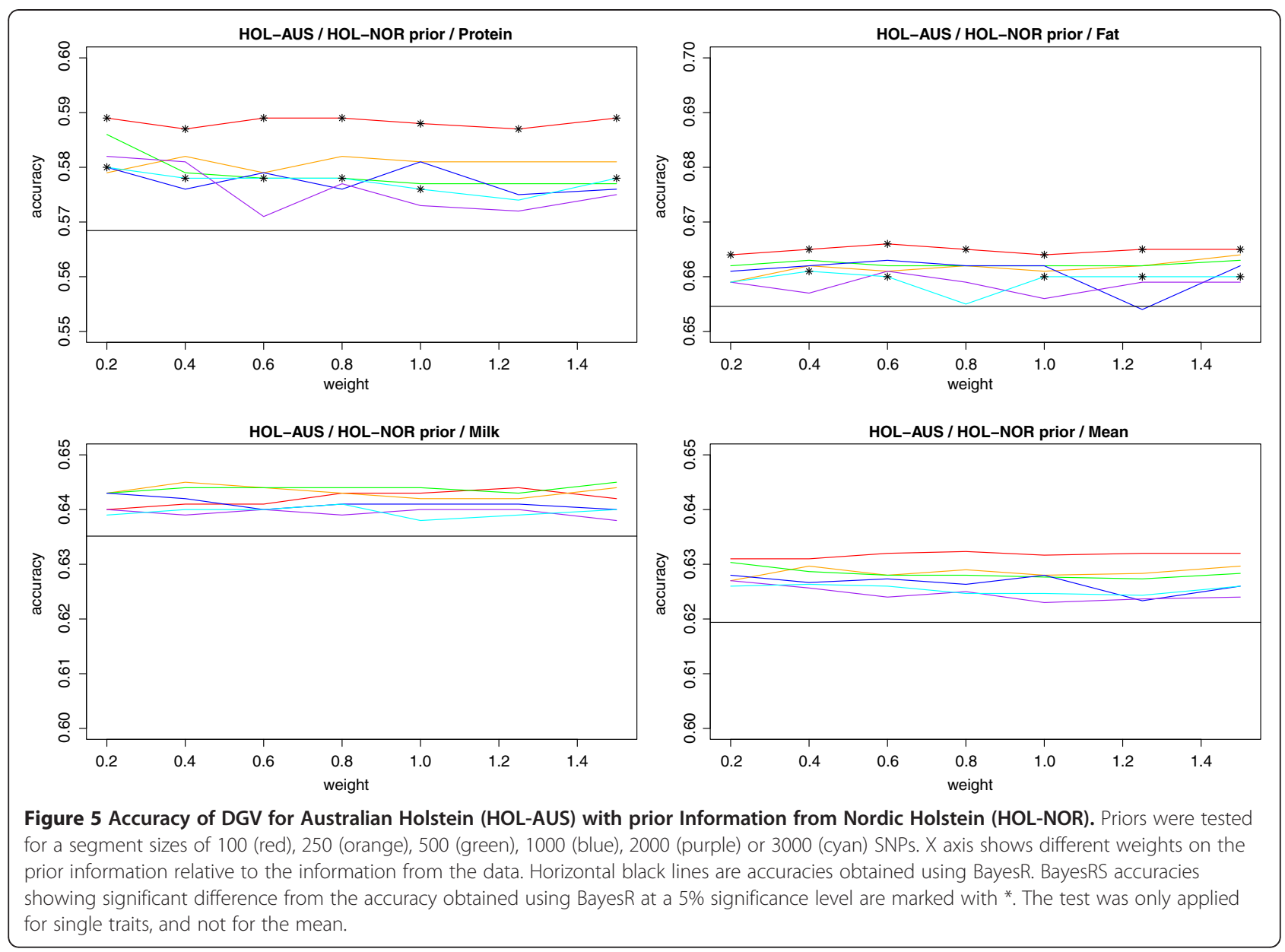

gave higher accuracies than a pooled reference for fat yield in JER-AUS. Further studies are needed to confirm the validity of the results in a larger validation population.

One advantage of the method presented here is a large reduction in computational demand. Since the BayesRS model only uses very condensed information from the other population, the increase in memory demand is negligible, and the extra complexity of the model only slightly increases the CPU run time. For JER-AUS running the BayesR model for 100.000 iterations required 33 hours, whereas the BayesRS model could be run for the same number of iterations in 39 hours. When using BayesR with the combined JERAUS HOL-AUS reference data, 100,000 iterations takes about 150 hours, and more than quadruples the memory requirements.

Although the accuracies obtained using BayesRS in most cases cannot compete with pooling of the data, the results seem consistently better than when using only data from the target population and a non-informative prior, for example only the JER-AUS data. In some cases where the extra data itself is not available, the BayesRS model or a similar approach could improve the accuracy of genomic predictions using only summary statistics. This might be in cases when intellectual property issues prevents sharing of the raw data, but allows use of summary statistics as in this study. The approach could also be useful for meta-analysis of many data sets from different sources.

The model presented here would also allow the use of other prior information such as known QTL or expression pathways, by assigning a higher prior probability to sample large effects in the involved genomic regions. In this study segments were chosen arbitrarily with a fixed length, but another approach could be to define coding and non-coding regions of the genome as different segments and set different Dirichlet priors. A challenge here would, however, be how to choose the counts in the Dirichlet prior without sampling them from a different population. Previous results show that SNPs near genes found in both human and bovine genomes are significantly associated with stature [12]. By considering evolutionary conserved regions as segments the method using external information sources presented in this 


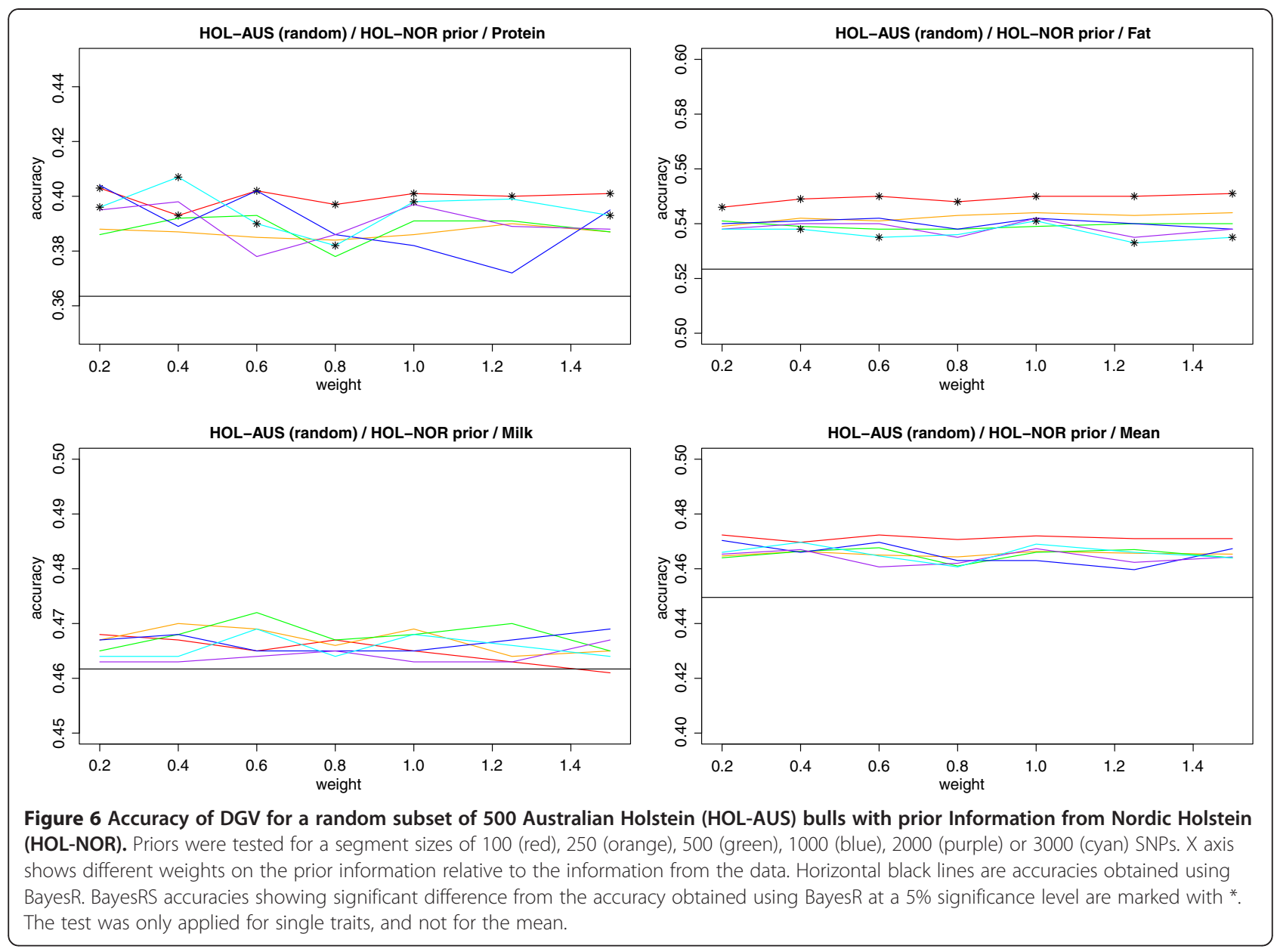

study could be used for genomic prediction across species for traits of common interest such as growth in meat-production animals or production traits in dairy species.

\section{Conclusion}

Our results suggest that genomic location specific priors in BayesRS improve the accuracy of genomic prediction, when the priors are derived from another population. However, the higher accuracies were only found to be significantly better than a competing alternative without location specific priors in a few cases. This might be a result of the limited number of animals used in the validation sets, so further investigation is needed to confirm the validity of the method.

Results also show that some highly variable segments coincide with known genes and QTLs, suggesting that using actual biological information could be beneficial for the accuracy of genomic predictions. Finally the BayesRS setup might offer a possibility for higher accuracies of genomic predictions in cases with limited computer resources or issues with data sharing.

\section{Competing interests}

The authors declare no competing interests.

\section{Authors' contributions}

Concept and design of the study was done by RFB, BJH, GS, MEG and MSL. The BayesRS model was developed by RFB, BJH and MEG. Implementation of statistical models in C++ was done by PJB. Analysis was carried out by RFB. The manuscript was drafted by RFB, BJH and GS. The final manuscript was read and approved by all authors.

\section{Acknowledgements}

We thank the Danish Cattle Federation (Aarhus, Denmark), Faba Co-op (Helsinki, Finland), Swedish Dairy Association (Stockholm, Sweden), and Nordic Cattle Genetic Evaluation (Aarhus, Denmark) for providing data. This work was performed in the project "Genomic Selection-From function to efficient utilization in cattle breeding (grant no. 3405-10-0137)", funded under Green Development and Demonstration Programme by the Danish Directorate for Food, Fisheries and Agri Business (Copenhagen, Denmark), the Milk Levy Fund (Aarhus, Denmark), VikingGenetics (Randers, Denmark), Nordic Cattle Genetic Evaluation (Aarhus, Denmark), and Aarhus University (Aarhus, Denmark).

\section{Author details}

${ }^{1}$ Centre for Quantitative Genetics and Genomics, Department of Molecular Biology and Genetics, Faculty of Science and Technology, Aarhus University, Tjele 8830, Denmark. ${ }^{2}$ Biosciences Research Division, Department of Primary Industries Victoria, Bundoora 3083, Australia. ${ }^{3}$ Land and Food Resources, University of Melbourne, Parkville 3072, Australia. ${ }^{4}$ Dairy Futures Cooperative Research Centre, Bundoora, Victoria 3083, Australia. ${ }^{5}$ La Trobe University, Bundoora, Victoria 3086, Australia. 
Received: 27 April 2012 Accepted: 5 October 2012

Published: 10 October 2012

\section{References}

1. Wray NR, Goddard ME, Visscher PM: Prediction of individual genetic risk to disease from genome-wide association studies. Genome Res 2007, 17:1520-1528.

2. Riedelsheimer C, Czedik-Eysenberg A, Grieder C, Lisec J, Technow F, Sulpice R, Altmann T, Stitt M, Willmitzer L, Melchinger AE: Genomic and metabolic prediction of complex heterotic traits in hybrid maize. Nat Genet 2012, 44:217-220

3. Goddard ME: Genomic selection: prediction of accuracy and maximisation of long term response. Genetica 2008, 136:245-257.

4. Lund MS, Roos APWD, Vries AGD, Druet T, Ducrocq V, Fritz S, Guillaume F, Guldbrandtsen B, Liu Z, Reents R, Schrooten C, Seefried F, Su G: A common reference population from four European Holstein populations increases reliability of genomic predictions. GSE 2011, 43:43.

5. Brøndum RF, Rius-Vilarrasa E, Stranden I, Su G, Guldbrandtsen B, Fikse WF, Lund MS: Reliabilities of genomic prediction using combined reference data of the Nordic Red dairy cattle populations. J Dairy Sci 2011, 94:4700-4707.

6. Hayes BJ, Bowman PJ, Chamberlain AJ, Verbyla K, Goddard ME: Accuracy of genomic breeding values in a multi-breed dairy cattle population. Genet Sel Evol 2009, 41:51.

7. Pryce JE, Gredler B, Bolormaa S, Bowman PJ, Egger-Danner C, Fuerst C, Emmerling R, Solkner J, Goddard ME, Hayes BJ: Short communication: Genomic selection using a multi-breed, across-country reference population. J Dairy Sci 2011, 94:2625-2630.

8. Matukumalli LK, Lawley CT, Schnabel RD, Taylor JF, Allan MF, Heaton MP, O'Connell J, Moore SS, Smith TPL, Sonstegard TS, Van Tassell CP: Development and characterization of a high density SNP genotyping assay for cattle. PloS one 2009, 4:5350.

9. de Roos APW, Hayes BJ, Spelman RJ, Goddard ME: Linkage Disequilibrium and Persistence of Phase in Holsten-Friesian, Jersey and Angus Cattle. Genetics 2010, 179:1503-1512.

10. de Roos APW, Hayes BJ, Goddard ME: Reliability of genomic predictions across multiple populations. Genetics 2009, 183:1545-1553.

11. Erbe M, Hayes BJ, Matukumalli LK, Goswami S, Bowman PJ, Reich CM, Mason BA, Goddard ME: Improving accuracy of genomic predictions within and between dairy cattle breeds with imputed high-density single nucleotide polymorphism panels. J Dairy Sci 2012, 95:4114-4129.

12. Pryce JE, Hayes B, Bolormaa S, Goddard ME: Polymorphic regions affecting human height also control stature in cattle. Genetics 2011, 187:981-984.

13. Browning BL, Browning SR: A unified approach to genotype imputation and haplotype-phase inference for large data sets of trios and unrelated individuals. Am J Hum Genet 2009, 84:210-223.

14. Kizilkaya K, Tait RG, Garrick DJ, Fernando R, Reecy JM: Whole genome analysis of infectious bovine keratoconjunctivitis in Angus cattle using Bayesian threshold models. BMC Proc. 2011, 5(Suppl 4):S22. Paris, France.

15. Dunn OJ, Clark V: Comparison of tests of the equality of dependent correlation coefficients. J Am Stat Assoc 1971, 66:904-908.

16. Grisart B, Coppieters W, Farnir F, Karim L, Ford C, Berzi P, Cambisano N, Mni M, Reid S, Simon P, et al: Positional candidate cloning of a QTL in dairy cattle: Identification of a missense mutation in the bovine DGAT1 gene with major effect on milk yield and composition. Genome Res 2002, 12:222-231.

17. Pryce JE, Bolormaa S, Chamberlain AJ, Bowman PJ, Savin K, Goddard ME, Hayes BJ: A validated genome-wide association study in 2 dairy cattle breeds for milk production and fertility traits using variable length haplotypes. J Dairy Sci 2010, 93:3331-3345.

18. Blott S, Kim JJ, Moisio S, Schmidt-Kuntzel A, Cornet A, Berzi P, Cambisano N, Ford C, Grisart B, Johnson D, et al: Molecular dissection of a quantitative trait locus: A phenylalanine-to-tyrosine substitution in the transmembrane domain of the bovine growth hormone receptor is associated with a major effect on milk yield and composition. Genetics 2003, 163:253-266.
19. Chamberlain AJ, Hayes BJ, Savin K, Bolormaa S, McPartlan HC, Bowman PJ, Van der Jagt C, MacEachern S, Goddard ME: Validation of single nucleotide polymorphisms associated with milk production traits in dairy cattle. J Dairy Sci 2012, 95:864-875.

20. Yang J, Manolio TA, Pasquale LR, Boerwinkle E, Caporaso N, Cunningham $J M$, de Andrade M, Feenstra B, Feingold E, Hayes MG, et al: Genome partitioning of genetic variation for complex traits using common SNPs. Nat Genet 2011, 43:519-U44.

doi:10.1186/1471-2164-13-543

Cite this article as: Brøndum et al:: Genome position specific priors for genomic prediction. BMC Genomics 2012 13:543

\section{Submit your next manuscript to BioMed Central and take full advantage of:}

- Convenient online submission

- Thorough peer review

- No space constraints or color figure charges

- Immediate publication on acceptance

- Inclusion in PubMed, CAS, Scopus and Google Scholar

- Research which is freely available for redistribution

Submit your manuscript at www.biomedcentral.com/submit 